Acknowledgement: This work was supported by the Institute for Rheumatic Research (IRR), Epalinges, Switzerland.

Disclosure of Interests: Raphael Micheroli: None declared, Amanda McGovern: None declared, Kerstin Klein: None declared, Xiangyu Ge: None declared, Paul Martin: None declared, Oliver Distler Grant/research support from: Prof. Distler received research funding from Actelion, Bayer, Boehringer Ingelheim and Mitsubishi Tanabe to investigate potential treatments of scleroderma and its complications, Consultant for: Prof. Distler has/had consultancy relationship within the last 3 years with Actelion, AnaMar, Bayer, Boehringer Ingelheim, ChemomAb, espeRare foundation, Genentech/Roche, GSK, Inventiva, Italfarmaco, iQvia, Lilly, medac, Medlmmune, Mitsubishi Tanabe Pharma, Pharmacyclics, Novartis, Pfizer, Sanofi, Serodapharm and UCB in the area of potential treatments of scleroderma and its complications. In addition, he had/has consultancy relationship within the last 3 years with A. Menarini, Amgen, Abbvie, GSK, Mepha, MSD, Pfizer and UCB in the field of arthritides and related disorders, Mojca Frank-Bertoncelj : None declared, Stephen Eyre: None declared, Caroline Ospelt: None declared

DOI: 10.1136/annrheumdis-2019-eular.4644

\section{SAT0056 TAS5315, A NOVEL BRUTON'S TYROSINE KINASE INHIBITOR, DEMONSTRATES POTENT EFFICACY AGAINST BONE DESTRUCTION IN AN ANIMAL MODEL FOR RHEUMATOID ARTHRITIS}

Naruto Noma, Fumihito Hosoi, Satoru Iguchi, Ryusuke Kaneko, Yohei Yoshiga, Yasunobu Arima, Daichi Akasaka, Kenji Tanaka, Akihiro Saito, Teruhiro Utsugi, Koichi Ikizawa. TAIHO PHARMACEUTICAL CO., LTD., Tsukuba, Japan

Background: Bone erosions and cartilage damages are a pathological hallmark of rheumatoid arthritis (RA) and are associated with poor functional outcome ${ }^{1}$. Aberrant activations of osteoclasts induced by receptor activator of nuclear factor $\mathrm{\kappa B}$ ligand (RANKL) $)^{2}$ are involved in the bone erosions of RA. It has also been recently shown that under chronic inflammatory conditions such as RA, inflammatory cytokines in joints induce pathological osteoclast differentiation and cause excessive bone resorption independent of RANKL-RANK signaling ${ }^{3}$. The Bruton's tyrosine kinase (BTK) signaling pathway plays a pivotal role in inflammatory response and bone resorption ${ }^{4}$. Thus, targeting BTK may be efficacious against not only inflammation but also bone erosion by regulating activation of effector cells such as B cells, macrophages and osteoclasts in RA. We have already shown the inhibitory effects of TAS5315 on RANKL-dependent osteoclast activation. However, it remained uncertain whether TAS5315 inhibits osteoclast activation induced by inflammatory cytokines.

Objectives: In this study, we evaluated the effects of TAS5315 on osteoclast activation induced by inflammatory cytokines (in vitro) and the infiltration of tartrate-resistant acid phosphatase (TRAP)-positive osteoclasts in the joint of a mouse CIA model.

Methods: In vitro biochemical assay was performed using available kinase assay panels. The BioMAP ${ }^{\oplus}$ Diversity PLUS panels were used to determine the profile of TAS5315 in primary human cell systems. The effects of TAS5315 on osteoclasts were assessed by examining osteoclast-mediated bone resorption. TAS5315 was orally administrated once a day in an established mouse CIA model. TRAP-positive osteoclasts were counted manually. Bone mineral density (BMD) and bone erosion were assessed using micro-CT analysis. The mechanical properties of the tibia were evaluated by a compression test of proximal metaphysis using a materialtesting machine.

Results: TAS5315 selectively inhibited the enzyme activity of BTK and had less off target inhibition against other kinases. In BioMAP ${ }^{\oplus}$ systems, TAS5315 decreased the production of $\mathrm{IgG}$ and the expression of cytokines (TNF- $\alpha$, IL-6, IL-17A). TAS5315 also inhibited osteoclast-mediated bone resorption induced by inflammatory cytokines. On the other hand, anti-RANKL antibody did not inhibit bone resorption induced by inflammatory cytokines. Futhermore, in the mouse CIA model, TAS5315 significantly ameliorated paw swelling and pathological changes. TAS5315 significantly decreased the infiltration of TRAP-positive osteoclasts in the joint and also showed improvement of BMD and bone erosion by timedependent micro-CT analysis. In vehicle-treated mice, the mechanical strength of tibia was decreased compared with normal mice. TAS5315treated mice recovered the decreased parameters of the mechanical strength compared with vehicle-treated mice.

These data suggests that TAS5315 improves bone erosion in murine model for RA through direct inhibition of osteoclast activation induced by inflammatory cytokines as well as RANKL.
Conclusion: Our study demonstrates that TAS5315 would be an attractive RA therapeutic drug that could improve bone destruction as well as inflammation

\section{REFERENCE}

[1] Nat Rev Rheumatol. 2012;8,656-64. 2: Nat Rev Rheumatol. 2015;11,18994. 3: Arthritis Rheumatol. 2016;68,2889-2900. 4: Drug Discov Today 2014;19,1200-4.

Disclosure of Interests: None declared DOI: 10.1136/annrheumdis-2019-eular.2019

\section{SAT0057 INCREASED MICRORNA-155 IS ASSOCIATED WITH A SPECIFIC DEFECT OF ANTI-INFLAMMATORY M2 MACROPHAGES POLARIZATION BOTH IN HUMAN RHEUMATOID ARTHRITIS AND IN COLLAGEN- INDUCED-ARTHRITIS MICE}

Audrey Paoletti ${ }^{1}$, Franceline Reynaud ${ }^{2}$, Julien Rohmer ${ }^{1}$, Juliette Pascaud ${ }^{1}$, Bineta Ly ${ }^{1}$, Elodie Rivière ${ }^{1}$, Nicolas Tsapis ${ }^{2}$, Elias Fattal ${ }^{2}$, Gaetane Nocturne ${ }^{1}$, Xavier Mariette'. ${ }^{1}$ Université Paris-Sud, INSERM U1184, Le Kremlin Bicêtre, France; ${ }^{2} 2$ Université Paris-Sud, Institut Galien, Châtenay-Malabry, France

Background: Monocytes/macrophages are key players in the pathogenesis of Rheumatoid arthritis (RA). Monocytes can differentiate into pro-inflammatory macrophages (M1-like) or into anti-inflammatory. macrophages (M2-like) We previously found a defect of monocytes polarization into M2-like macrophages, in RA patients and not in healthy donors (HD) or in patients with other inflammatory diseases (such as connective tissue diseases or spondyloarthritis), and a propensity for preferential maturation towards M1 like pro-inflammatory macrophages that could contribute to synovial inflammation.

MiR-155 expression has been shown elevated in RA synovial macrophages and in blood monocytes. MiR-155 inhibits mRNA of 2 important proteins involved in programming of M2 macrophages (SOCS-1 and C/EBP- $\beta$ ).

Objectives: We assessed if miR-155 could play a role in the defect of monocytes polarization into M2-like macrophages both in human RA and in a mouse model of collagen-induced-arthritis (CIA) mice.

Methods: Monocytes were isolated from PBMCs for HD and RA by negative selection. Purified monocytes were transfected using AMAXA technologies with miR-155 mimic (for HD) or antagomiR155 (for RA) or controls miRs. Then monocytes were incubated in the presence of human serum (SAB) (M2-like) for 6 days. Expressions of total macrophages markers (CD11b and CD71), M2-like macrophage markers (CD163 CD206 and Arg-1), M1-like macrophage marker (iNOS) were evaluated by flow cytometry, ELISA or immunofluorescence.

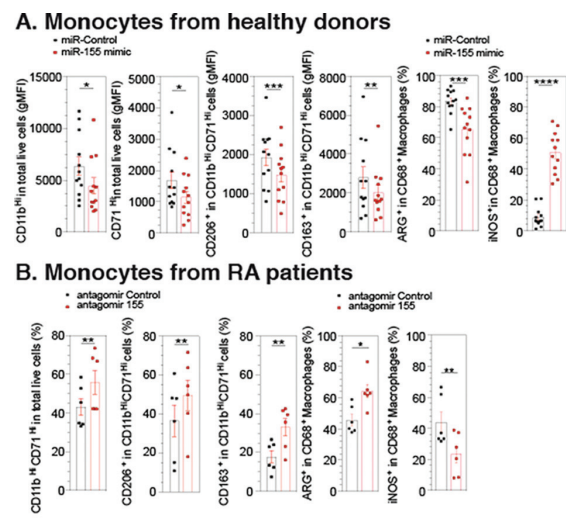

\section{Monocytes from CIA or control mice}

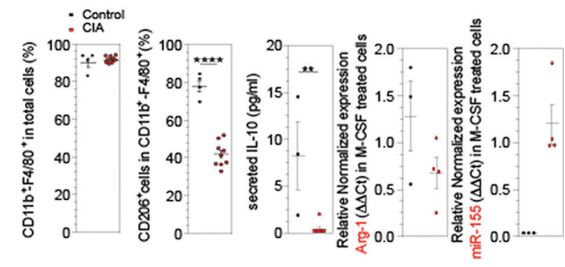

